\title{
Industry 4.0, transition or addition in SMEs? A systematic literature review on digitalization for deviation management
}

\author{
Zuhara Chavez $^{1}$ D . Jannicke Baalsrud Hauge ${ }^{2} \cdot$ Monica Bellgran $^{1}$
}

Received: 18 May 2021 / Accepted: 15 October 2021 / Published online: 7 November 2021

(c) The Author(s) 2021

\begin{abstract}
Nowadays, Industry 4.0 (I4.0) has become a trendy topic in manufacturing industries worldwide. The definition is far from being comprehensible for small players, and the practical uptake is ambiguous. Transnational companies are often at the top in deploying I4.0 features, learning primarily from their experimentation. Alternatively, small- to medium-sized enterprises (SMEs), given their less stable value chains and unsteady processes, tend to target most of their efforts on controlling disturbances and adopting solutions for deviation control. Such solutions can be features that set the path for SMEs to transition to I4.0. This paper aims to examine the reported degree of digitalization in implemented solutions in SMEs when handling deviations and analyze the integration of such solutions in their digital transformation process. Systematic literature review (SLR) is used to examine literature published up to and including January 2019. The results show a higher concentration on practical applications rather than on frameworks. Existing frameworks that focus on SMEs address particular elements of I4.0 rather than a gradual shift with a holistic view, increasing the deployment difficulty for SMEs. This paper identifies potential constraints in deployment if such a trend maintains for consecutive years.
\end{abstract}

Keywords Deviation management $\cdot$ Digitalization $\cdot$ Industry 4.0 $\cdot$ SME $\cdot$ Systematic review

\section{Introduction}

The early interpretation of the I4.0 paradigm dealt with "smart manufacturing" (SM), putting the factory at the center, imagining its transformation through digitalization. The "real-time capability" is an enabling 4.0 fundamental principle, creating the condition for design processes to be constantly informed by data from internal functions, networks, and customers and elaborated and visualized in real-time [1]. I4.0 is a paradigm shift from "centralized" to "decentralized" production by technological advances, a setback of conventional production process logic. I4.0 provides an entirely different industry approach to manufacturing with new technologies to maximize output while minimizing resource utilization [2]. It is foreseen that I4.0 will revolutionize the way the design, manufacture, operation,

Zuhara Chavez

zuhar@kth.se

1 Department of Sustainable Production Development, Production Management, Royal Institute of Technology, Södertälje, Sweden

2 Department of Sustainable Production Development, Production Logistics, Royal Institute of Technology, Södertälje, Sweden service, and consumption of products are done due to the high mass customization that will be enabled $[3,4]$.

The competitive nature of today's industry forces more factories to move toward implementing high-tech methodologies [5]. For instance, by employing the Internet of Things (IoT) and Cyber-Physical Systems (CPS), "sensing enterprises" turn into a reality. Manufacturers can sense deviations from the production plan when they appear and identify delays in the logistics network in real time. Nevertheless, a significant issue remains in the digital, sensing, and smart factory of the future, i.e., how digital integration and real-time data monitoring can be realized in practice $[6,7]$. Self-organization and self-optimization are aspects typically subsumed under the term I4.0. Existing integration solutions represent complex and centrally organized systems, which are costly to adapt and maintain. Thus, they prohibit the flexible integration of IT systems and the self-organization of manufacturing processes, especially in small- to medium-sized enterprises (SMEs) with a limited budget of resources and limited IT knowledge [8]. SMEs tend to struggle to adapt to external market forces and make the next step in expanding their business due to a lack of expertise and resources $[9,10]$. 
According to the analysis presented by Maier [11], the countries where SMEs have been most successful in adopting new technologies have coherent government strategies in place to support them, for instance, Germany (Industrie 4.0), China (Made in China 2025), and the USA (America Makes). It has resulted in higher productivity levels in these countries over others with lower levels of adoption. Gomes, Rigley, Bacon, et al. [9] state that if cloud manufacturing (CM) follows this example, there needs to be a robust and coherent strategy to advise SMEs on how best to utilize $\mathrm{CM}$ for their needs. Deviation management is of particular importance for SMEs. SMEs are highly dependent on maintaining their capacity to respond to their clients' needs while maintaining a competitive advantage in their market. To have a fast response while covering business requirements, SMEs need continuous effort in improving their management processes, from planning until evaluating their operational performance [12, 13]. Disturbances can often hinder the response capacity in the production system that leads to deviations, putting the production system performance at risk. Digitalization is shifting the way production systems are managed; however, reducing production disturbances and deviations is still a high priority for production management.

The concept of deviation is often seen with the quality management perspective and analyzed under statistical control, looking into the variation in the quality specifications. Likewise, Juran and Godfrey [14] state that any significant deviation from the normal pattern has a cause that, once determined, can give an idea of the variability in the production processes. In this, we refer to deviation as "a result that does not conform to the established or accepted standard." Deviations can be caused by internal and external undesirable events and setbacks, defined as "disturbances" $[15,16]$. A disturbance causes deviation in the system performance, causing a loss in production. The principle is that production disturbances should be measured as deviations from the normal, desirable circumstance [17]. Some examples of common disturbances in SMEs are changed orders from customers, material shortages, machine failures, absenteeism, and tool shortages, to mention some. Such disturbances can lead to deviations that hinder to a great extent the company's performance in terms of efficiency, productivity, or delivery to customers. Disturbances are part of day-to-day operations in SMEs [15], where deviations' effects impose a challenge on the robustness of the production system, i.e., the ability to absorb manufacturing disruptions without failing [18], and defy the implementation of I4.0.

Researchers project that smaller companies will struggle due to the high investments needed. In contrast, the better flexibility that I4.0 provides could lead prominent players to steal market segments now usually dominated by SMEs, e.g., customized products [19]. Nonetheless, if it is anticipated that SMEs will fall behind, there is a need to devote research that supports and helps the small players to carry on and adapt to the shift.

Despite the few but emerging frameworks about I4.0 and conceptual articles on the research area, to the best of our knowledge, it has not been studied how SMEs integrate digitalization when handling deviations and how they can utilize current efforts as a turning point toward adopting the I4.0 paradigm. There is a growing interest, and extensive reviews on I4.0 continue emerging [2, 10, 13, 20-23]; however, these systematic reviews do not integrate deviation management as a critical element in the search terms.

The main objective of this article is to explore the status of research in the area, identifying existing knowledge in terms of frameworks and practical applications captured in literature. It is intended to grasp the current research condition by identifying the gaps and weaknesses and setting an outline for future research that will guide SMEs toward a successful I4.0 journey. Therefore, our focus is on the digitalization degree of practical applications supporting deviation management and integration in SMEs' digital transformation journey.

The paper is organized as follows. Section 2 gives the background of the study in terms of the gap. Section 3 describes the study's objective and the research method, while Section 4 presents the results from the SLR. Section 5 contains the findings classified into five different research blocks. Section 6 presents a discussion in terms of four research gaps. Lastly, Section 7 ends the paper with a conclusion, recommendations for researchers and SME managers, and the limitations of the study.

\section{Theoretical background}

Despite all the emerging research around I4.0, the terminology around the research area is still young. It lends itself to confusion, as there is no generally accepted understanding of the I4.0 concept being issued [19, 20, 23]. For instance, it could refer to both intelligent upgrades and the concepts of "I4.0," "Smart Manufacture," and "Industrial Internet of Things" (IIoT). The acatech (German academy of science and engineering) model [24] and the RAMI (reference architectural model) framework [25] provide a general starting approach for grasping the concepts, giving a perspective on the essential aspects in I4.0, however, at a higher and abstract level for SMEs.

Academic and consulting studies focus on multinational enterprises; often, they treat SMEs similarly in their evolutionary path toward SM or Industry 4.0. Nevertheless, given SMEs' distinct differences and requirements, this can be considered challenging [10]. Furthermore, given no consolidated perspective on the term I4.0, the practitioners' 
criteria for deciding whether the company has implemented intelligent upgrades may vary from person to person [26]. For instance, different entities could refer to I4.0 to allude to different smart solution applications or implementation; an entity can be considered a country, company, or organization. Still, the fourth industrial revolution is characterized by self-awareness and autonomy. The factory should collect data about its elements, i.e., people, machines, and environment of the factory system, and self-assess, taking its own decisions (autonomy) [27].

The I4.0 transformation is driven by nine foundational technology advances: (1) autonomous robots, (2) simulation, (3) horizontal and vertical system integration, (4) the industrial internet of things, (5) cybersecurity, (6) the cloud, (7) additive manufacturing, (8) augmented reality, and (9) big data and analytics, according to Rüßmann, Lorenz, Gerbert, et al. [4]. In the I4.0 paradigm, elements like sensors, machines, and IT systems are linked along the value chain, i.e., further than a single enterprise. These connected systems (also called cyber-physical systems) can act together using standard Internet-based protocols and analyze data to predict failure, configure themselves, and adapt to changes. All upstream manufacturing processes (such as R\&D, sourcing, and inbound logistics) and downstream functions (such as outbound logistics, retail, and customer services) should be included within the 4.0 architectural model [1]. It has not been fully exemplified to what extent SMEs are deploying the nine blocks of I4.0 and how to achieve the whole connection of systems in such a context.

Manufacturing environments are globalized, enterprises are highly pressured to increase their competitiveness to deal with dynamic and turbulent markets, and developing an intelligent manufacturing system has been identified as an effective way to increase the enterprise's competitiveness. One way to see or define the level of intelligence of a manufacturing system is to consider the number and types of "smart things" within the system. Therefore, an irreversible trend in evolving manufacturing systems is to make as many "smart" manufacturing resources as possible to leverage system adaptability and resilience for uncertainties and changes [28]. There is a challenge in finding a standard definition of "intelligence" and "smart" and determining intelligence levels in a system. For instance, process deviations can happen during production, leading to a non-executable process, i.e., nonstandard situations. In this scenario, the deviation might stop the production process, and problems emerge in manufacturing operations. The production system has to react accordingly [16] to prevent the problem from escalating. Likewise, the level of intelligence can be connected to the ability to deal with deviations while minimizing the impact on performance.

There are multiple definitions of the concept of I4.0; for that reason, it is crucial to set a boundary to ensure validity.
We limited the scope of the study to the definitions presented by previous works $[13,20,29]$, unifying the perspectives in a single concept:

a new approach for controlling and improving production processes by ensuring real-time harmonization of flows, automated data collection, and analysis, assisting the customized manufacturing of products and allowing the collaboration between processes, products, suppliers, and customers through the internet.

Although significant effort has been made to conceptualize the terms I4.0 and smart technologies [30] and empirical works $[5,9,31,32]$, the reality of SMEs' positioning in such a setting is not yet clear.

\section{Objective and method}

This paper aims to find out the research coverage in terms of smart solutions and digitalization in SMEs under the umbrella of I4.0. In this context, the study seeks an answer to two main research questions:

RQ1: What is the degree of digitalization in solutions for deviation handling in SMEs?

RQ2: How could SMEs integrate such solutions in their digital transformation journey?

The study utilized SLR as the primary method and backward snowballing as a secondary search strategy to be as comprehensive as possible. Therefore, it required detailed work on the queries definition and rigorous evaluation of the terms to ensure the search was widespread without being vague or general; given the context of the topic, it could quickly become overly broad. To reduce human error, the authors eliminated the manual data coding and extraction by employing NVivo 12 as a support resource, whereas other research papers on literature reviews manually utilize spreadsheets. The paper provides an overall picture of the studies reported as scientific articles in major journals covering the research area of production and operations management, specifically deviation handling and digitalization in SMEs.

\subsection{Systematic literature review as a method}

SLR aims to produce a protocol that does not compromise the researcher's creativity in the review while still reducing the bias of traditional narrative reviews. SLR offers comprehensive sources and a clear search strategy. As Cook, Mulrow, and Haynes [33] state, high-quality systematic reviews can define the boundaries between what is 
known and what is not known and can support us to avoid knowing less than what has been proven.

The present study employs an SLR strategy based on the one proposed by Smart, Tranfield, and Denyer [34]. The method follows a four-stage protocol: planning the review, conducting the review, a report, and dissemination; this sequence has been utilized by other researchers in the field $[13,35,36]$. We analyzed the mentioned research publications in which they applied the method to seek lessons learned and best practices from SLR deployment. SLR secures coverage by using search strings in reliable databases that can be replicated. Ensuring coverage while allowing replication is a precondition when performing a literature review to eliminate errors and bias.

The goal for the review presented by Smart, Tranfield, and Denyer [34] focused on using the SLR as it is used in medical science. Their SLR focuses on the management research-social science field, employing the evidencebased approach for creating management research that is rigorous and relevant to the practitioner community. The four stages reviewed and included in this SLR are as follows:

1. Planning the review. Select the population or sample, i.e., focus of the study. This phase aims to produce a protocol that does not compromise the researcher's creativity in the literature review process, while also reducing the risk of researcher bias, as traditional narrative reviews.

2. Conducting the review. This stage involves four identified critical steps.

i. Identify keywords and search terms (from a scoping study) from the literature review and discussion with a team. Determine the search strings that are more appropriate for the study. Specify in detail to allow replication.

ii. Search published journals, bibliographic databases, unpublished studies, conference proceedings, industrial trials, the Internet, and even personal requests.

iii. Provide a complete list of results (articles and papers) on which the review will be done. Follow the sequence: (a) determine inclusion and exclusion (here, more than one reviewer should assess if there are disagreements the review panel can solve); (b) relevant sources are submitted for a more detailed evaluation of the full text; (c) choose items for a systematic review. Document all the sources included and excluded with reasons, then evaluate quality studies, where a checklist can be employed, for example [37]. An assessment can consist of a primary marker, sampling strategy, data quality, theoretical adequacy, and generalizability.

iv. Construct data extraction forms, which reduce errors. They can be constructed as paperbased or computer-based. From them, it is necessary to build summary tables for data synthesis. Consist of details of the information source (title, authors, journal, and publication details) and any other study features such as population characteristics, the context of the study, and an evaluation of the study's methodological quality.

3. Reporting. Report the findings using thematic analysis, identifying critical emerging terms/topics and research questions. Outline what is already known and established from the extraction forms in the previous step.

4. Disseminating. Link themes across the various core contributions wherever possible. Highlighting such links is an essential part of the reporting process. Perform a thematic analysis, map what is already known, what elements have a consensus, and detect emerging topics. The reviewer should provide a detailed audit trail back to the core contributions to justify and ground their conclusions. The study must encourage questions from reviewers.

The snowballing approach is complementary to database searches. It allows the researcher to identify relevant articles that may not necessarily appear in a database search using a search string [38]. The starting point is the initial list of relevant articles selected as applicable in the area. The backward approach consists of scanning through the referenced articles in the initial list. Based on the defined inclusion and exclusion criteria, new papers are selected. The final inclusion decision is given after reading the full pre-selected papers.

\section{Systematic literature review on digitalization for deviation management in SMEs}

The SLR four-stage protocol inspired by Smart, Tranfield and Denyer [34], and replicated by selected works in the field [13, 35, 36], was described in chapter 3 . The study progression of the four stages-(1) plan the review, (2) conduct the review, (3) report, and (4) disseminate-is presented in the following sections. 


\subsection{Stage 1: plan the review}

Stage 1 comprehends planning the SLR population and determining the study sample, which is the focus of the study. This paper emphasizes deviation management in SMEs, and intends to study the knowledge around recognizing, analyzing, and synthesizing how SMEs handle deviations, in the form of digitalization or by using any other solutions. The databases employed in the SLR are Scopus, Web of Science, Emerald Insight, and EBSCO; these sources are recognized for containing sustainable publications on production operations, management, and technologies. The initial draft of the planning phase included the databases Springer, Primo, and IEEE. However, the authors removed those for two principal reasons: (1) low search results or (2) database restrictions in the number of possible terms for query construction. The articles were categorized and arranged using the software NVivo 12 as a support tool, for coding the literature and as a substitute for relying only on spreadsheet forms for data extraction and classification of qualitative data. Mendeley Desktop 1.19.3 software served as a support tool for reference management.

\subsection{Stage 2: conduct the review}

Setting inclusion and exclusion rules for a particular study was essential to conducting the review. In this case, the search was limited to peer-reviewed academic journals, conference articles, or book sections fully available. The period covered January 2010 up to and including January 2019, considering only research with full content published in the English language. In the first general screening, the search terms were title, keywords, and abstract.

Three main search phrases and terms were identified deviation management, smart solutions, and digitalization. Alternative and associated terms were established to expand the search. Likewise, truncation and wildcards were applied. The three search phrases and terms necessary to narrow the results to the interest areas and research questions were I4.0, SMEs, and sustainability. The identified six terms were used to create six different categories that allowed searches to be performed in blocks determined by a mix of the search terms. The principal author selected Scopus as the initial database to test the search terms with the completed list of terms. The same author, acting as a reviewer, checked the search terms against a thesaurus to ensure that the spelling was accurate and included similar options. After this first check by one reviewer, the word groups were discussed with the rest of the authors, acting as a review panel, following the recommendation of the SLR methodology authors [34] and the lessons learned by studying review papers in the field.
From the review of the complete preliminary list of search terms, the terms and phrases "production operations," "production management," "manufacturing," and "operations" were included to narrow down the sources to research that was specific to the area. The addition of these terms originated in category 1.1, as indicated in Table 1 . The last set of search terms included were sustainability and the circular economy concept; both were incorporated to identify emerging research in the field. The assumption was that no hits would appear when referring to the mentioned terms combined with SMEs due to the topic being in its early stages (low maturity).

The queries defined in the categories followed an incremental sequence; each search string included all the related terms of each set of words or phrases, as indicated in Table 1, i.e., seven search strings were tested in four different databases, where the last search string included the terms from categories 1 to 6 including 1.1.

\subsection{Stage 3: report}

Following the search queries, the results obtained are presented in Table 2. The hits from categories 1 to 3 set the scene of the focus area; we observed the search results to be of high volume. However, to reach this SLR's objective, it was necessary to delimitate the search with the terms in categories 4, 5, and 6 . The detailed screening started from category 4 to category 6 with 290 papers identified, indicating the need to refine the results by following inclusion and exclusion rules.

The selection process is detailed in Fig. 1, starting with 290 papers from the four different databases. The results were assessed on the research trend and type of research method; titles were scanned to detect any potential article focused on SMEs that may have appeared before the following categories. The analysis on the title, keywords, and full text started with categories 5 and 6 with 48 papers, removing duplicates and applying inclusions and exclusions with the following criteria:

- NE: All papers in non-English were eliminated from the databases that allowed language as a filter from the beginning. English was set at the start of the search.

- NT: Text not fully available.

- NR: Not related to I4.0 and deviation management.

After screening using the coding criteria and eliminating duplicates, only 16 papers met all the requirements. The search results of each database from this screening are summarized in Table 3 . The remaining 16 papers' full text were analyzed and assessed, and qualitative and quantitative studies were included. A complete list is presented in 


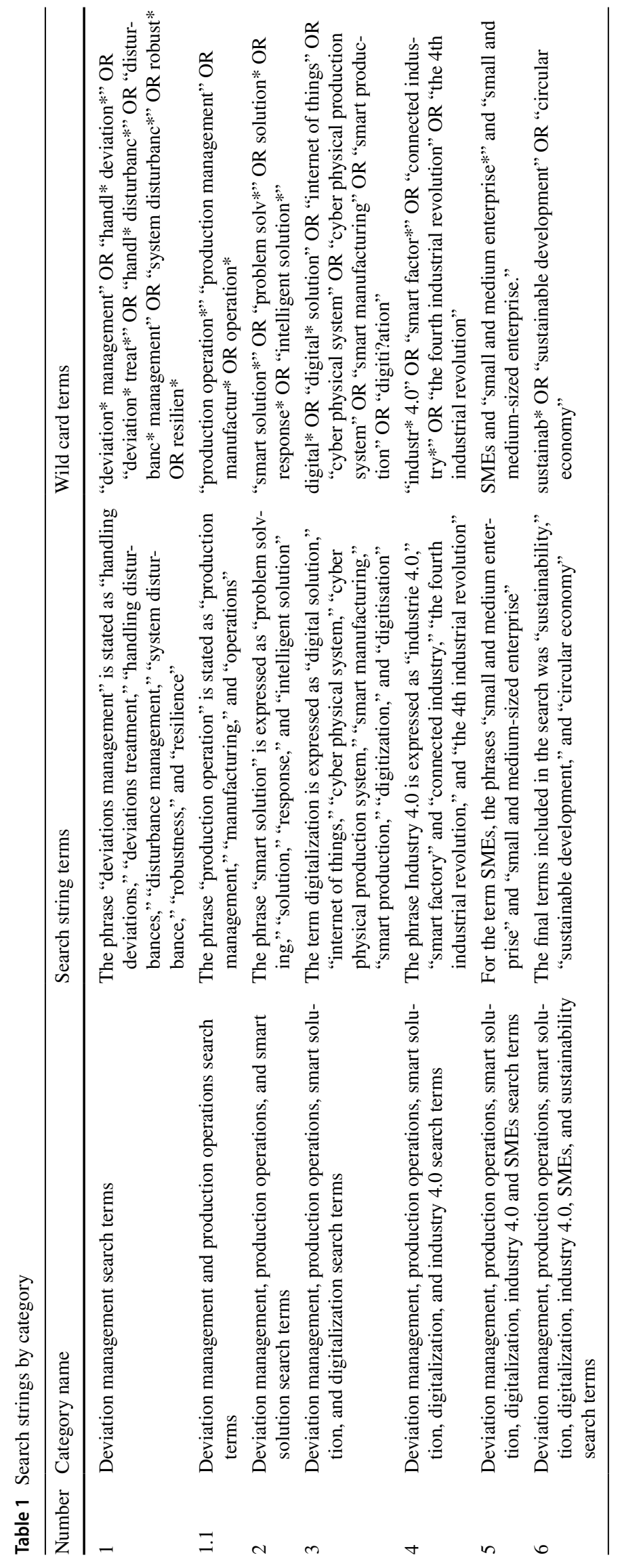


Table 2 Search results by databases

\begin{tabular}{lllll}
\hline Categories & Scopus & Web of Science & EBSCOhost & Emerald Insight \\
\hline 1 & 92318 & 522641 & 47441 & 33340 \\
1.1 & 80000 & 51131 & 6584 & 21917 \\
2 & 39628 & 17444 & 2089 & 19457 \\
3 & 6911 & 1163 & 49 & 3209 \\
4 & 178 & 33 & 0 & 31 \\
5 & 19 & 3 & 1 & 9 \\
6 & 10 & 0 & 0 & 6 \\
\hline
\end{tabular}

Table 4, indicating each paper's categorization, which is further explained in stage 4.

\subsubsection{Snowballing strategy}

From the selected papers listed in Table 4, the authors applied a snowballing backward approach to complement the SLR to ensure relevant papers were included and to compensate for the limiting adopted approach in the SLR search strategy. The references in each article included in the initial SLR results list were scanned, resulting in 14 papers to review. Each paper was analyzed following the inclusion and exclusion criteria. Table 5 lists the 8 papers that were selected after evaluating the full texts, fulfilling the same criteria as the database search.

The selected 24 papers place from 2015 to 2019 . Figure 2 reveals the scarcity of research and its rise from 2018, with significant growth from the previous year. Even though our SLR included only January in 2019, we observe an increase compared to earlier years. The results' trend shows an interest in developing further knowledge that supports SMEs on their journey toward I4.0.

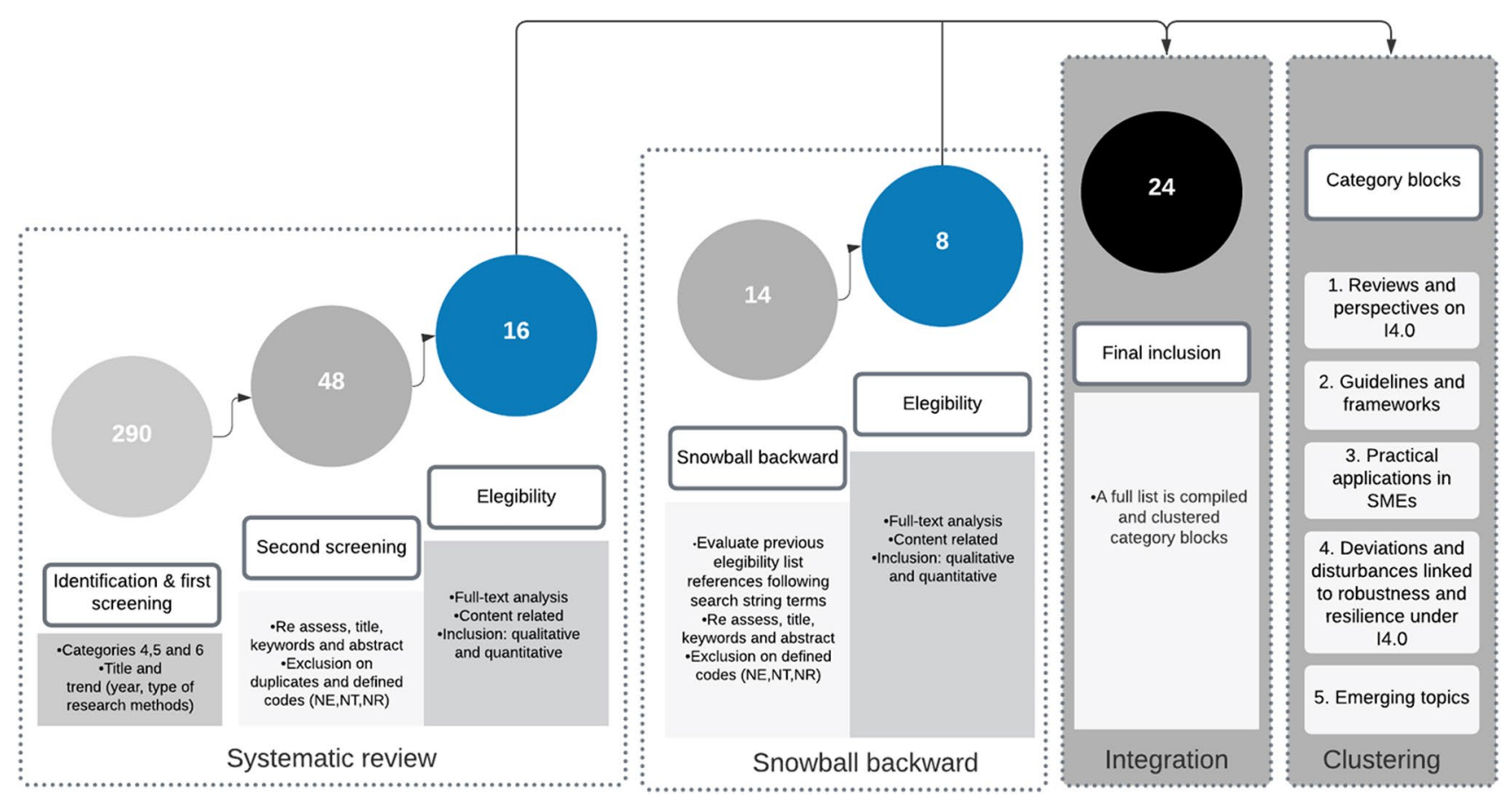

Fig. 1 Screening process

Table 3 Screening summary based on coding criteria, duplication, and exclusion

\begin{tabular}{|c|c|c|c|c|c|c|}
\hline \multirow[b]{2}{*}{ Database } & \multicolumn{3}{|c|}{ Category 5} & \multicolumn{3}{|c|}{ Category 6} \\
\hline & Total & $\begin{array}{l}\text { Duplication and } \\
\text { Exclusion }\end{array}$ & Inclusion & Total & $\begin{array}{l}\text { Duplication and } \\
\text { Exclusion }\end{array}$ & Inclusion \\
\hline Scopus & 19 & 5 & 14 & 10 & 10 & 0 \\
\hline Web of Science & 3 & 1 & 1 & 0 & 0 & 0 \\
\hline EBSCOhost & 1 & 1 & 0 & 0 & 0 & 0 \\
\hline Emerald Insight & 9 & 8 & 1 & 6 & 6 & 0 \\
\hline
\end{tabular}


Table 4 Summary of systematic literature review, initial screening

\begin{tabular}{|c|c|c|c|c|}
\hline Author(s) & Block & Database & Publication date & Description \\
\hline (Lee et al., 2016) [31] & 1 & Scopus & 2016 & Framework and architecture of cyber manufacturing \\
\hline (Bertola and Teunissen, 2018) [1] & 1 & Emerald Insight & 2018 & $\begin{array}{l}\text { Understanding I4.0 implementation, implications, and link to } \\
\text { sustainability }\end{array}$ \\
\hline (Wang et al., 2019) [26] & 1,3 & Scopus & 2019 & $\begin{array}{l}\text { Factors affecting the intelligent upgrades of SMEs and policies to } \\
\text { promote the intelligent upgrades }\end{array}$ \\
\hline (Lee et al., 2015) [5] & 2 & Scopus & 2015 & Developing and deploying a CPS for manufacturing \\
\hline (Mukhopadhyay et al., 2019) [27] & 3 & Scopus & 2019 & $\begin{array}{l}\text { AOI (automatic optical inspection) based IC detection on PCB, } \\
\text { algorithm evaluation }\end{array}$ \\
\hline (Bi et al., 2018) [28] & 3 & Scopus & 2018 & $\begin{array}{l}\text { Conceptual design of sensors and instrumentations, level of intel- } \\
\text { ligence }\end{array}$ \\
\hline (Ud Din et al., 2018) [39] & 3 & Scopus & 2018 & $\begin{array}{l}\text { Agent-oriented smart factory, I4.0 implementation architecture } \\
\text { for SMEs }\end{array}$ \\
\hline (Veres et al., 2018) [30] & 3 & Scopus & 2018 & $\begin{array}{l}\text { Supply chain optimization by black hole algorithm, smart solu- } \\
\text { tion toward Industry } 4.0\end{array}$ \\
\hline (Wieland et al., 2016) [8] & 3 & Scopus & 2016 & $\begin{array}{l}\text { Manufacturing-integration assistant “MIAlinx," IF-THEN mod- } \\
\text { eling rules, smart solution }\end{array}$ \\
\hline (Dallasega et al., 2017) [7] & 3 & Web of Science & 2017 & I4.0 approach to ETO (engineer to order) plant-building industry \\
\hline (Boorla et al., 2018) [40] & 4 & Scopus & 2018 & Manufacturing strategies for robustness in I4.0 \\
\hline $\begin{array}{l}\text { (Martínez-Olvera and Mora- } \\
\text { Vargas, 2018) [18] }\end{array}$ & 4 & Scopus & 2018 & Robust systems and resilience of a production system in I4.0 \\
\hline (Morisse and Prigge, 2017) [41] & 4 & Scopus & 2017 & $\begin{array}{l}\text { Resilience in Industry } 4.0 \text {, model to describe challenges in } \\
\text { Industry } 4.0\end{array}$ \\
\hline (Gomes et al., 2018) [9] & 5 & Scopus & 2018 & $\mathrm{CM}$ for a sustainable manufacturing, methods, and limitations \\
\hline (Dubey et al., 2019) [42] & 5 & Scopus & 2019 & $\begin{array}{l}\text { Data analytics capability, organization flexibility, supply chain } \\
\text { resilience, and competitive advantage }\end{array}$ \\
\hline (Moreno and Charnley, 2016) [3] & 5 & Scopus & 2016 & $\begin{array}{l}\text { Circular economy and re-distributed manufacturing toward } \\
\text { Industry } 4.0\end{array}$ \\
\hline
\end{tabular}

Table 5 Summary of the literature review from backward snowballing approach

\begin{tabular}{|c|c|c|c|}
\hline Author(s) & Block & Publication date & Description \\
\hline (Ramírez-Correa et al., 2019) [43] & 1 & 2019 & Impact of innovativeness and social influence on performance expectancy in SMEs \\
\hline (Varaniute et al., 2018) & 2 & 2018 & $\begin{array}{l}\text { Preconditions of adopting IoT in SMEs and resulting business model transforma- } \\
\text { tions }\end{array}$ \\
\hline (Stock and Seliger, 2016) [44] & 3 & 2016 & $\begin{array}{l}\text { Retrofitting of manufacturing equipment to monitor the existing operational states } \\
\text { of the equipment }\end{array}$ \\
\hline (Jones et al., 2019) [45] & 3 & 2019 & $\begin{array}{l}\text { Collection of a volume of data in real-time leading to rapid analysis at the machine } \\
\text { level, and auto-evaluation of I4.0 aspects }\end{array}$ \\
\hline (Wang et al., 2016) [46] & 3 & 2016 & $\begin{array}{l}\text { Cloud computing technologies (CCT) to share computing resources and support } \\
\text { advanced features in human resource management }\end{array}$ \\
\hline (Bi et al., 2015) [47] & 3 & 2015 & $\begin{array}{l}\text { Illustrate the importance of redesigning, reusing, remanufacturing, recovering, } \\
\text { recycling, and reducing (6R) for sustainable manufacturing and discuss the } \\
\text { procedure to reconfigure robots }\end{array}$ \\
\hline (Bi et al., 2014) [48] & 5 & 2014 & $\begin{array}{l}\text { A new perspective of economically evolving dedicated machines or manufacturing } \\
\text { systems into sustainable systems }\end{array}$ \\
\hline (Garcia-Muiña et al., 2018) [49] & 5 & 2018 & $\begin{array}{l}\text { Introduction of the principles of sustainability and transition from a linear to a } \\
\text { circular economy }\end{array}$ \\
\hline
\end{tabular}

\subsection{Stage 4: dissemination}

The analyzed articles are displayed in Fig. 3, classified under five different category blocks, following a logical categorization according to the encountered research streams in literature. The five blocks are defined as (1) reviews and perspectives on I4.0, (2) guidelines and frameworks, (3) practical applications in SMEs, (4) deviations 
Fig. 2 Papers' distribution over five years (2015-2019)
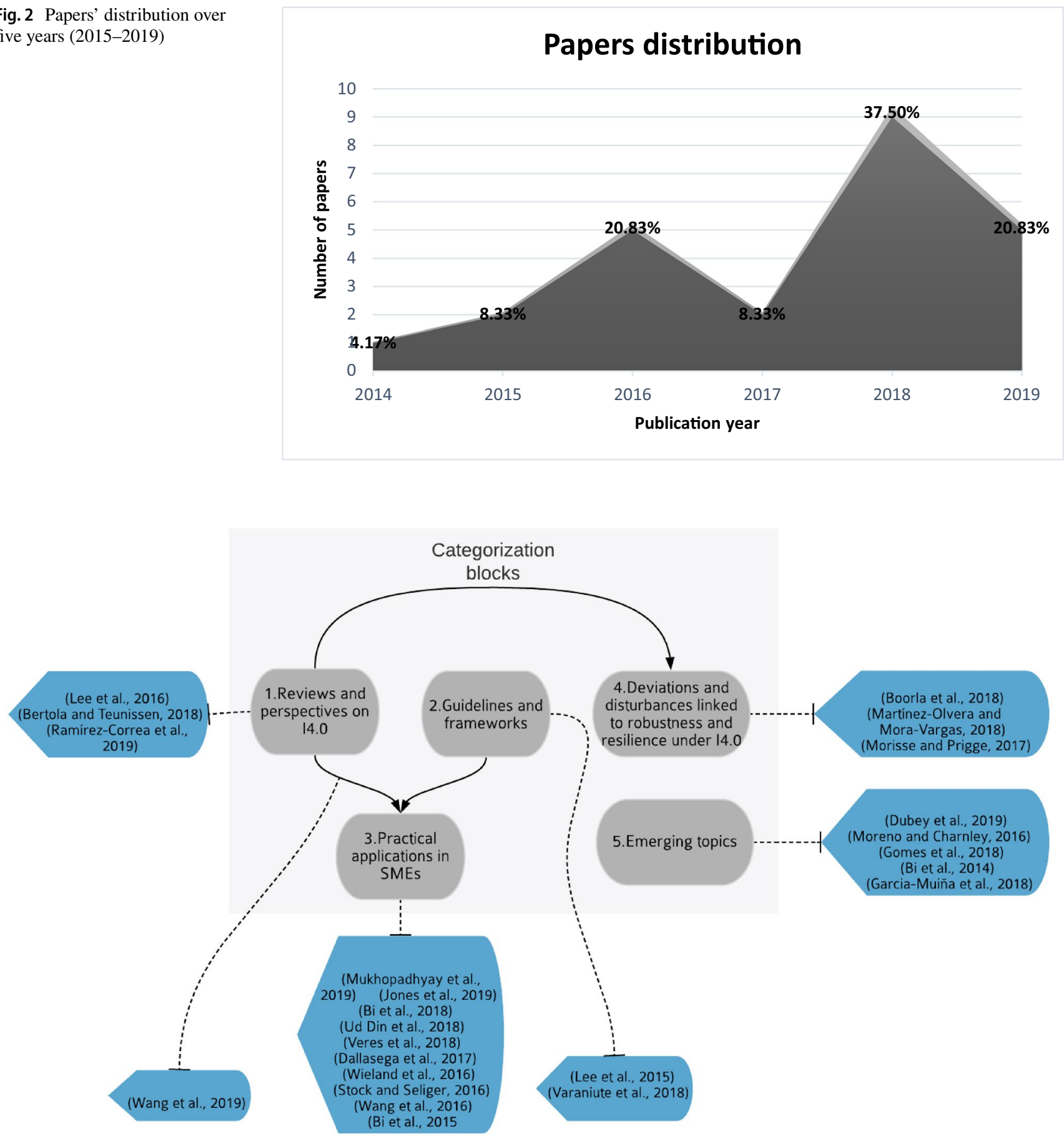

Fig. 3 Category blocks from the systematic literature review

and disturbances linked to robustness and resilience under I4.0, and (5) emerging topics. Practical applications form the most significant block, showing that higher efforts on developing knowledge for SMEs are put on applied concepts or tools that have been pre-tested in other scenarios, i.e., leading companies in technology, transnationals, or OEMs. Disturbances and deviations were not directly linked to the applications in block 3 , exemplified neither conceptually nor explicitly, but appeared when talking about robustness, challenges, and resilience for I4.0. Hence, such publications were classified together in block 4 . Figure 3 contains details of the five categorization blocks and interconnections. The different works in each block are described in Tables 4 and 5 . 


\section{Findings from the literature review}

The infant state of the research area can be stated as the reason why guidelines for implementation are scarce. Guidelines to implement I4.0 are emerging [50]. They have been assessed by other literature reviews, for example, when integrating I4.0 and in lean manufacturing [20, 51]. However, the scenario is different when discussing frameworks and guidelines for I4.0 implementation in SMEs. Previous systematic literature reviews have presented efforts to support SMEs. Those works have identified targeted performance objectives, described the required managerial capabilities and technologies for each case [13], analyzed I4.0 maturity models and their characteristics, and identified requirements for SMEs [10]. Despite those efforts in the area and several autonomous frameworks under the umbrella of I4.0, there are few widely acceptable implementation frameworks specifically for SMEs [10, 39]. The research works found in the SLR show dedication to particular elements of I4.0 as an approximation to full implementation, i.e., e-manufacturing, cyber manufacturing, cyber-physical systems, and CM.

\subsection{Reviews, perspectives, guidelines, and frameworks for industry $\mathbf{4 . 0}$ implementation}

The closest framework found in the SLR results is Lee, Bagheri, and Kao [5], presenting a guideline for implementing CPS in a 5-level architecture guideline. The proposed 5-level CPS structure, namely the 5C architecture, provides a step-by-step guide for developing and deploying a CPS for manufacturing applications. It provides a comparison of today's factory and an I4.0 factory, where the main attributes are differentiated (e.g., lean operations: work and waste reduction vs. worry-free productivity). It explains the process of implementing CPS in today's factories. The framework offers several advantages that can be categorized into three stages: (1) component, (2) machine, and (3) production system. It emphasizes the gradual upgrading from the current position. Later work [31] introduces a cyber-manufacturing fundamental framework and architecture compared to e-manufacturing. Challenges identified were lack of standards, handling of big data, and cybersecurity. It visually shows the connections between big data analytics and cyber manufacturing systems, comparing different e-manufacturing and cyber manufacturing characteristics.

The results on reviews of 14.0 showed a couple of works $[1,43]$. Bertola and Teunissen [1] provide insights into the I4.0 framework and principles, aiming to show their impacts on business units, processes, and components within the fashion industry context. The expert's perspective is enriched by several descriptive case studies as exampled. The article shows possible trajectories, enabling an effective transformation of the textile and apparel industry through the deployment of the I4.0 paradigm. It provides an outline of the implications of I4.0 for this sector, which can be extrapolated to other sectors. This research illustrates how a proper digital transformation and suitable implementation can reshape industries into more sustainable and customer-oriented companies. Furthermore, it critically addresses how the most prominent fashion leaders are slow in adopting new technologies and provides a clear overview of the gradual evolution of the fashion industry by adopting new technologies. Bertola and Teunissen's [1] work makes evident the necessity for a framework that serves as a blueprint to guide efforts for SMEs and leaders in different industries; therefore, its selection in this study is logical.

The work of Ramírez-Correa Grandón, Arenas-Gaitán, et al. [43] claims to be the first paper to analyze the impact of innovativeness and social influence on performance expectancy by those responsible for information and communication technologies in SMEs in a developing country. The study focused on Chilean SMEs following a descriptive analysis. The random sample analysis indicated that IoT use is low. The lack of IoT adoption in developing countries makes managers of SMEs, and people in general, ignore the usefulness of IoT technologies.

The authors aimed to validate a research model that explicates the performance expectancy of IoT from cognitive and psychological variables, which we did not find in other papers in this SLR. The contribution of this study lays on increasing knowledge about the adoption of IoT, particularly in developing countries. Something that stands out in this paper is that it explores social influence as a significant antecedent of the behavioral intention of technology when trying to understand SMEs' technology adoption. The study's results indicate that the cognitive variable, social influence, psychological variable, and personal innovativeness of information technology are relevant to explaining IoT's performance expectancy.

\subsection{Digital applications in SMEs: current state toward Industry 4.0}

The practical application studies comprise the largest category block in the SLR analysis. By practical applications, we refer to specific solutions presented in the selected papers. All the eligible research works in this block linked their applications to I4.0 and highlighted low cost as a primary driver and strength factor [7, 8, 26-28, 30, 39, 44-47]. Table 6 presents an overview of the papers in this section, 
Table 6 Key characteristics in applications per study

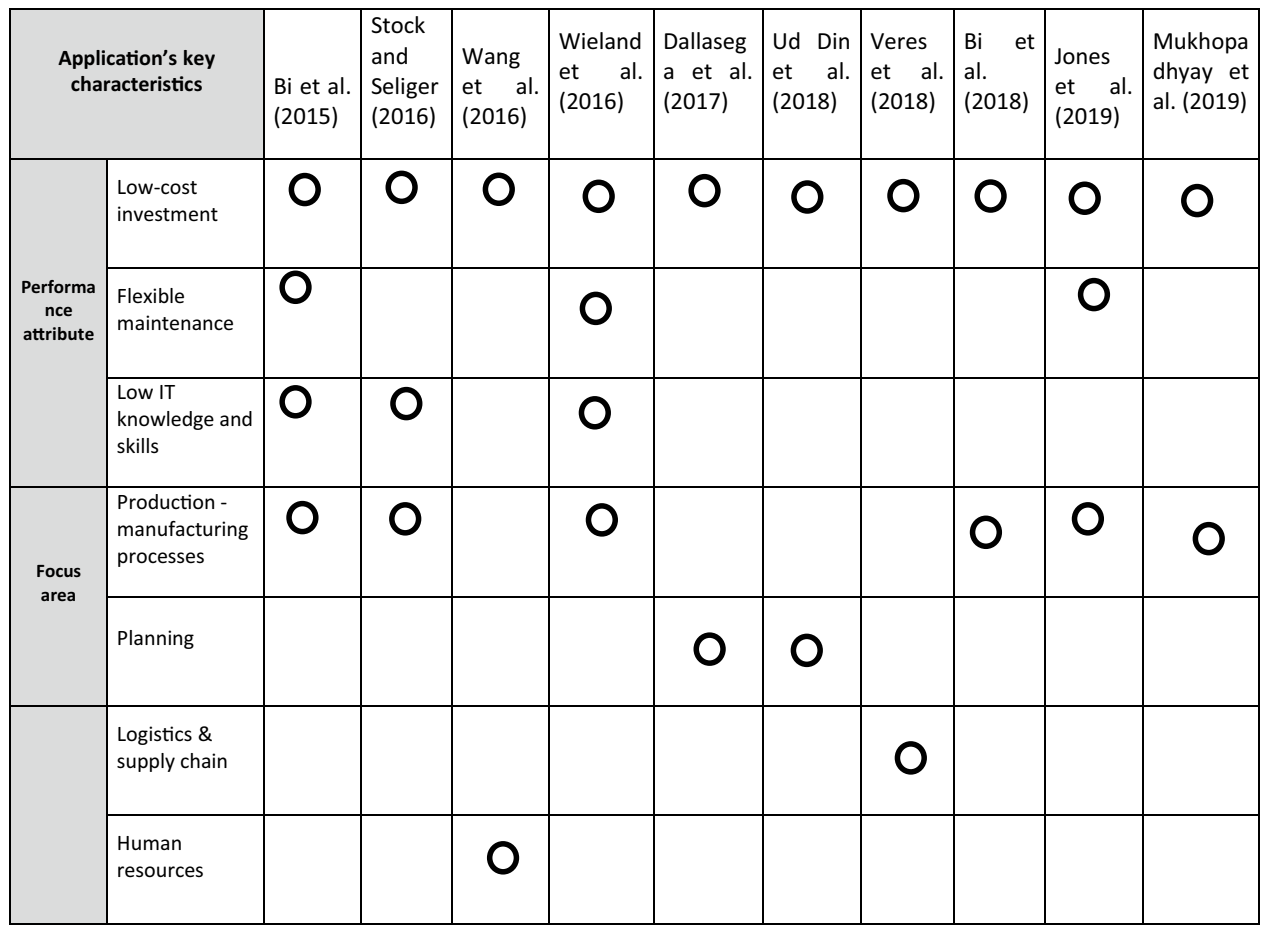

referring to the key characteristics, breaking down the performance attributes of the application and the application area, i.e., production or manufacturing processes, planning, logistics and supply chain, and human resources.

In response to RQ1, we could infer that in most assessed cases, the digitalization state seems to be at an early stage; in each work, the focus of the application emerged in response to the company's intention to update its systems to comply with I4.0 tendencies. Jones, Zarzycki, and Murray [45] is the only work that intentionally assesses the overall understanding of the concepts and technology applications under I4.0. In Table 6, the performance attribute can provide a rough overview of the degree of digitalization of smart solutions in SMEs; low-cost investment is a shared concern among the different cases. The selected papers do not necessarily refer to decreased in production disturbances or better handling deviations by the created solutions. The closest to making this connection is Stock and Seliger [44], where the presented solution allowed for reporting causes of machine failures; for that reason, we included in Table 6 classification of the focus area, which can provide a broad indication of the type of disturbances that can potentially be impacted, i.e., decreased or better managed.

For instance, the works focusing on production and manufacturing processes presented solutions that (a) aimed to decrease processes' variability by replacing manual inspection with automatic visual inspection [27]; (b) improved measurement systems by using measurement sensors that can be adaptable to changes and uncertainties automatically [28]; (c) improved the monitoring of production processes by better production data acquisition and maintenance with a manufacturing-integration assistant [8]; and (d)improved machine speed to perform a high-quality leak test [45].

The need to assess specifically intelligent upgrades was highlighted by Wang, Shou, Wang, et al. [26]. Given that previous research had a narrow focus, i.e., transforming and updating or productivity and performance, the degree of "intelligence" in the upgrades was not assessed. It is the only paper that focused on evaluating the degree of digitalization as "intelligence" in the SME upgrades; it has not been compared to the rest of the papers in Table 6 because it does not present a solution, but an assessment and critical points in regard to the updates performed by SMEs. However, it agrees with the rest of the works compared in Table 6 regarding the low-cost investment factor. Given its contribution, this study is visualized in Fig. 3 as a connection between block 1 and block 3. Wang, Shou, Wang, et al. [26] considered the difficulty of financing as a significant problem that hinders SMEs from upgrading or adopting technologies. Thus, it is a crucial factor assessed in their algorithm. They also studied the factors affecting the intelligent upgrades of SMEs to provide empirical evidence for further promoting intelligent upgrades of SMEs. Their suggestions mentioned adopting different policies for different countries and industries to promote intelligent upgrades of SMEs. This type of study in other countries can serve as a starting point to push for real upgrades in SMEs. Previous studies on knowledge management in SMEs [36] have also pointed out policy design and policy initiatives as an 
essential factor for encouraging knowledge and innovation, which is associated with growth.

In their paper, Mukhopadhyay, Murthy, Arora, et al. [27] proposed AOI (automatic optical inspection) for PCB inspection problems in SM. They evaluated their algorithm on different PCBs, and a detailed comparison study is described in various lighting conditions and with different types of cameras. Since the solutions are aimed at SMEs, solutions with expensive machine vision cameras are not affordable.

Bi et al. [28] analyzed the requirements of dynamic measurements of gripping forces and introduced a conceptual design of sensors and instrumentations for grippers. Their motivation was to observe the very few cost-effective solutions available for measuring the gripping force of a robotic gripper. It gives a good introduction to the lack of low-cost solutions for making companies and production systems smart. Wieland, Hirmer, Steimle et al. [8] present an approach for realizing a rule-based manufacturingintegration assistant called "MIAlinx." It aims to benefit SMEs on the production floor and in their journey toward I4.0. It connects sensors and actuators based on defined rules that people in the organization, such as production workers without extensive experience in IT, can define and use. Future work is encouraged on an advanced method for easy modeling rules and practical testing to determine the complexity of the rules, optimizations, and evaluation. Such application works raise the need for low-cost solutions in implementation and maintenance for SMEs [8, 27, 28].

A unique work that shows how an SME auto-evaluated aspects related to the Industry 4.0 definitions is Jones, Zarzycki, and Murray [45]. They dissected the various core concepts and then expanded the process to cover the nine pillars on which I4.0 is built. The auto-assessment was performed against each significant element, seeking whether the concepts and requirements were understood inside the organization. They also looked at the challenges I4.0 poses for the future of their business. The on-site application of the I4.0 nine technological advancements was assessed, affirming that the SME already deals with many of the attributes covered by I4.0 definitions. The SME in this study stated an increase in requests from their customers to use more expensive and sophisticated control systems capable of processing large amounts of data within the machine and producing the data for MES systems. The lack of adequate skill sets and the reluctance to change are continuous challenges for the SME; acknowledging it is essential to nurture an environment open to new ideas, new technology, and change. Practical examples are given; for instance, a recent project is a high-speed machine to perform a high-quality leak test on a medical device. It involves collecting a volume of data in real-time, leading to its rapid analysis at the machine level and autonomous decisions.
An innovative strategy to enhance central manufacturing resources' utilization rates, utilizing modular architecture and redesigning, reusing, remanufacturing, recovering, recycling, and reducing (6R) practices comes from $\mathrm{Bi}$, $\mathrm{Liu}$, Baumgartner, et al.'s [47] study. They illustrated the importance of $6 \mathrm{R}$ for sustainable manufacturing and reflect on the general procedure of robot reconfiguration. Flexibility and cost are two critical challenges in adopting industrial robots in SMEs, given that the same type of task can be limited because of the size of the SME. The 6R processes allow a robot to adopt new jobs, increase its utilization rate, and reduce unit costs of products. This work stated clearly that in the implementation, a universal solution is not available. The solutions have to be tailored to specific SMEs, and should vary over time.

A study by Stock and Seliger [44] presented a use case built on sustainable development toward I4.0 using an information and communication technology (ICT) infrastructure; a retrofitting solution for a desktop machine tool developed in a laboratory of sustainable manufacturing in a research center. The retrofitting solution was supposed to monitor the existing operational states of a milling machine, i.e., shut on/off, idling, processing, and fault. The definition of the monitoring strategy comprised defining the measuring parameters, the monitoring position, the sensor orientation, the sensor application, and the execution of the measurement. Connected to a smart product, the retrofitted machine could decentrally schedule the material flow and automatically react to any machine failures by, for instance, notifying the responsible worker. A significant aspect to highlight is the fact that this machine could now be implemented in a CPS. The solution was designed to be easy to install and cost-effective.

Works focusing on planning, with gradual application, were presented in Ud, Henskens, Paul, and Wallis [39] and Dallasega, Rojas, Rauch, and Matt [7]; both studies made it clear that the coverage of their application was limited and needed further deployment at the factory level. Ud, Henskens, Paul, and Wallis [39] presented a scheme for implementing I4.0 in SMEs called AOSF (agentoriented smart factory framework). The AOSF framework had two objectives; first, to provide an architecture for SMEs, including end-to-end supply chain integration in compliance with I4.0 standards. Second, to provide intelligence and decision-making at the base level through an AOSR-WMS (Agent-Oriented Storage and RetrievalWarehouse Management System) planner. The AOSRWMS and framework were tested in a warehouse; their future work needs to handle tasks with the same priority to provide more flexibility in decision-making at the user side. Implementing the plant side and multiple dimensions of the user side was also intentionally left for an upcoming development in this particular project. Dallasega, Rojas, 
Rauch, and Matt [7] proposed an ICT-supported, nearly real-time-capable production planning approach, which utilized simulation and showed a drastic reduction in the on-site inventory level. The new pull model reduces the average buffer content by approximately $30 \%$ vs. working on the pull scheme. I4.0 principles, like "real-time capability," "decentralization," and "self-control," are the leading enablers to obtain lean, agile, and responsive production systems in the engineer to order-plant (ETO-plant) building and construction industry. Further work on real-world data to validate the model is necessary. Nevertheless, the application is shown as an example of an early step toward I4.0 in the construction sector.

The only work that goes slightly further is Veres, Illes, and Landschutzer [30], which focused on the supply chain and compared algorithms for speed and accuracy. The efficiency of both algorithms was demonstrated with a supply chain optimization problem in the automotive industry. The application of this paper was in the automotive industry. Even though it highlighted the industry and showed a clear connection to I4.0 already done by other authors [52-54], it is the only one that dedicated effort into assessing the programing characteristics required.

Decision-making activities in a manufacturing system rely significantly on information systems. According to Wang, Wang, Bi et al. [46], the emerging CCTs have been proven as both opportunities and challenges for SMEs; they proposed to use them to share computing resources and support advanced features with a focus on the applications in SMEs. Their work is the only one in the SLR results that has human resources as the focus area. Examples of what can be achieved with the application are tasks within (a) training and development, where the developed model enterprise can tailor employees' training and development plans to the required qualifications at work; (b) employee recruitment; and (c) performance management, where it is possible to quantify the strategies of SMEs and the performance management also provides quality indicators for employee assessments. The proposed information system enhances the system performance of traditional human resource activities. It expands the system's flexibility to deal with uncertainties and changes and makes processes standardized.

The degree of digitalization of each application is hard to assess, as there is no joint agreement on the desired entire state that complies with I4.0. Further empirical research is needed both in the development and in the deployment of applications and studies on how the adoption of I4.0 elements influences the deviation management in SMEs. The results lack a clear link on disturbances effects with the applications or features acquired by the SMEs in the mentioned studies.

\subsection{Deviations and disturbances linked to robustness and resilience under the Industry 4.0 paradigm}

The works from the search results displayed production disturbances, and deviations were assessed in papers that addressed resilience and robustness. The studies contained in this category block addressed the factors from different perspectives. For instance, Martínez-Olvera and MoraVargas [18] examined variation under disturbance propagation and effects; Boorla, Eifler, McMahon, and Howard [40] centered on evaluating the variation and its adjustability, while Morisse and Prigge [41] focused directly on resilience and the characteristics necessary to build it up. Boorla, Eifler, McMahon, and Howard [40] highlighted the principle of adjustability as one aspect that complies with I4.0 demands when focusing on proactive communication. Deviation was mentioned only as a variation in the physical product specification related to quality and design. This manufacturing strategy for robustness applies to any product and process. The authors state that higher measurement frequency leads to accurate estimation.

Martínez-Olvera and Mora-Vargas [18] studied the propagation of disturbances, utilizing the Max-Plus algebra approach as a modeling tool to investigate the propagation of manufacturing disturbances. The study made a clear distinction between resilience and a robust system. It highlighted the objective of systems for I4.0, which are intended to be robust, i.e., can absorb manufacturing disruptions without failing or breaking. Seven diverse scenarios were examined, consisting of varying processing times. The findings demonstrate that the primary disturbance effect is primarily enclosed in the immediate elements, independent of the processing time variation origin. The subsequent disturbance effect initially increases (as it continues spreading) and then disappears. Additional research is needed to determine under which conditions a resource will become critical to set appropriate robustness strategies. Morisse and Prigge [41] addressed resilience in I4.0, conducting a literature review and qualitative study. According to them, six characteristics are necessary to achieve resilience in I4.0: flexibility, diversity, connectivity, knowledge, redundancy, and robustness. They developed a model called a "resilience house," which aims to acquire these characteristics. This research paper highlighted challenges for organizations in the evolution of I4.0; nonetheless, it is necessary to test the model to evaluate its practicability. Resilience was underlined as a competitive approach, and robustness was mentioned as one of the resilience characteristics. Overall, they emphasized the importance of analyzing each organization to define the target and roadmap.

One common factor presented in the studies in this block is the variation analysis under production processes and 
operations; all the authors in the papers included in this category agreed that it is necessary to control and fix variation for reducing disturbances and deviations. Nevertheless, the views were limited to product variation, which is far off from complete integration with I4.0. Resilience characteristics may be identified in works like Morisse and Prigge [41], but how exactly SMEs can achieve this is far from being uncovered. Future studies should explore and scrutinize the characteristics of the practical side to ensure that companies can build resilience on concrete theoretical bases and differentiate what is suitable for SMEs to start with.

\subsection{Emerging topics}

The terms sustainability and circular economy were actively included in category 6 to detect the emerging research connecting sustainability with I4.0. Five studies addressed the terms; given the tendency, we assumed that performing the search with an extended time frame (beyond January 2019) would increase the results for this category. Gomes Rigley, Bacon, et al. [9] linked CM with sustainability by identifying four fundamental methods of how CM increases sustainability: (1) collaborative design; (2) greater automation; (3) increase in process resilience; and (4) enhanced waste reduction, reuse, and recovery. Bi, Pomalaza-Ráez, Singh, et al. [48] built on a new perspective of system reconfiguration by redesigning, reducing, reusing, and recycling (4R) existing manufacturing resources. Moreno and Charnley [3] linked the circular economy with re-distributed manufacturing for a resilient system, while Garcia-Muiña, González-Sánchez, Ferrari, and Settembre-Blundo [49] proposed that circular economy is a possible economic model. Additionally, two topics that appeared in the selected works were re-distributed manufacturing and data analytics. Dubey, Gunasekaran, Childe, et al. [42] explained how data analytics play an essential role in organizational competitive advantage and resilience. Fields such as re-distributed manufacturing and circular economy are still in their infancy, and the available literature is disjointed and have multiple perspectives [3]. Gomes, Rigley, Bacon, et al. [9] aimed to demonstrate how $\mathrm{CM}$ offers a more sustainable manufacturing future to the industry.

As resource scarcity grows and climate change disrupts global supply chains, there will be an ever-increasing need for manufacturing processes that utilize waste valorization strategies rather than relying on fresh material and energy inputs. CM provides opportunities for manufacturers to improve sustainability as a model for implementing a circular economy approach. The authors highlight the struggle of SMEs in adopting new technologies due to a lack of expertise and resources. CM is an example of new technology that can support SMEs to overcome those hurdles. Moreno and Charnley [3] focused on two main concepts and their relation, circular economy and re-distributed manufacturing. This paper aims to scrutinize re-distributed manufacturing as a concept and the opportunities to make possible resilient consumption and production systems over circular innovation. They present I4.0 as a clear strategic vision of the capabilities of digital aptitude to enable redistributed manufacturing. The paper defines some of the challenges in the future for attaining re-distributed and circular manufacturing.

The work of Dubey, Gunasekaran, Childe, et al. [42] set out to theoretically and empirically establish the linkages among data analytics, supply chain resilience, and competitive advantage. Their work focused on data quality and analysis rather than on specific disturbances. Disturbances were analyzed on two spectrums: one as events such as natural disasters and the other as disruptions in the supply chain, e.g., political crisis, strikes, or fire in the plant; building theory on a different perspective, i.e., beyond production processes, compared with the works included in previous sections. The study attempted to explain how data analytics capability under the moderating effect of organizational flexibility improved supply chain resilience and competitive advantage. They suggested that organizations increase their data analytics capability by investing in vertical information systems to improve supply chain resilience and competitive advantage.

A case study [48] introduced a new perspective on system reconfiguration, i.e., recycling an obsolete test machine to meet new functional requirements, showing a significant economic benefit. This case study provided a unique perspective on economically evolving dedicated machines or manufacturing systems into sustainable systems. The new view was based on redesigning, reducing, reusing, and recycling (4R) existing manufacturing assets. The advantage of implementing such a strategy is that a system configuration can still be designed as a dedicated system with a minimal set of prerequisite functions to meet current customers' needs. Once some changes are enacted, the concept assumes that system components would be systematically considered for reuse and expanded to accommodate the changes.

Building on sustainability improvement, Garcia-Muiña, González-Sánchez, Ferrari and Settembre-Blundo's [49] study verified that a circular economy is a possible economic model that goes beyond mere business metrics and implies significant changes, essential not only within the companies that want to equip themselves with this model but also in the relations between the different stakeholders. The authors stated that the proactive approach to sustainability must go beyond simple compliance with environmental regulations, which means changing processes and vision, transforming the company from the ground up, for instance, through business model innovation, to integrate innovation and sustainability as a strategic competitive advantage. 
The author's statement is that technological innovation is complemented by organizational innovation, meaning that a change for competitiveness can create value other than economic growth.

The studies in the emerging topics block open new research areas that must be considered to further develop the concept of I4.0 to improve organizations' operations and overall sustainability. The current efforts originate from relations between data analytics, I4.0, and sustainability; their operationalization needs further and dedicated research to present frameworks that facilitate SMEs transitioning to digital and sustainable ways of working.

\section{Discussion of the research gaps}

This section aims to answer the research questions by assessing particular gaps encountered in the analysis in Section 5. The answers and gaps are framed based on the literature and the authors' analysis.

\subsection{Research gap 1: understanding what the data implications are for solutions for deviation management in SMEs}

The data quality and quantity are two traits that increase the I4. 0 challenge, as meaningful information must be inferred from decision-making data; the same goes for deviation handling. The data quality and quantity are highlighted as potential drawbacks by different publications in this SLR [5, 31, 42]. These connect to data accessibility, accuracy, and reliability as aspects that companies must take care of to develop accurate and intelligent decision-making support systems $[55,56]$. To our surprise, such data aspects were not present in the studies on practical applications in SMEs. The new elements introduced in such applications most likely will also generate data that can effortlessly increase the complexity of the current data inventory. Before starting with any application or digital solution deployment, it will be valuable to analyze what a company already possesses in terms of data, where it is contained, and how to utilize it further for the desired business objectives.

Handling data will be a challenge for organizations transitioning to digital ways of working [5, 41]. By 2011, McKinsey estimated that manufacturing had the most significant data stored annually [57]. Meanwhile, the volume, velocity, and variety of the generated data have given industries a noticeable challenge: how to extract actionable information from this big data. Machine analytics must be effectively integrated with factory operation analytics [31] to address data issues like extraction and conversion. Most manufacturing companies are still at the beginning of collecting and using data gathered from production processes, dealing with the problem that significant parts of the collected data are outdated or biased and not in real time. Often the big shift for SMEs is transitioning from handling their production planning with spreadsheets to a formal ERP system. I4.0 technologies offer the opportunity to simplify the data gathering by providing accurate information and precise feedback for reliable production planning and control [7]. There are currently several tools and methodologies available for converting data into information, yet the organization must analyze the converted information to gain value. Such information can be linked to decision-making for deviation management, and the added value can, for instance, offer prioritization criteria, support designing contingency plans, or provide early warnings for faster responses.

Additionally, in an I4.0 context, companies are encouraged to share data, and have open access, joint projects, and shared collaborations. Some questions that arise, how do companies handle the data that is being generated? Moreover, how to categorize the data and support the conversion into information while preserving security inside organizations. To achieve efficient data handling and conversion securely, suitable digital tools are needed [30]. Cybersecurity is one of the significant hurdles in implementing cyber manufacturing, as it is critical to have resilient capabilities for connected machines and systems in a cloud environment [31]. Manufacturers are naturally reluctant to share their data with competitors. Wang, Wang, Bi, et al. [46] give an example of how to build a data-sharing architecture at an SME level; however, a framework needs to be developed to allow the manufacturing cloud to act as a third party to utilize another company's manufacturing data and models without disclosing this information to the competing company [9].

\subsection{Research gap 2: establishing the connection between digital solutions for SMEs and 14.0 frameworks}

In answer to RQ1, none of the applications in block 3 highlights the degree of digitalization in the implemented solutions, even though they connect the theoretical background and motivation to the I4.0 paradigm. Their solutions highlight the benefits in terms of the technical problem and emphasize low-cost implementations. There is also a lack of connection to a planned journey or complete digital transformation, which implies that SMEs are interested in starting the transformation without understanding the further implications for an entire digital shift. Since there are no precise standardization levels published that can be generalized, it is challenging to assess the degree of digitalization in solutions for deviation management. There is also a connection missing in the practical application cases with deviation management. A good approximation can be understanding the type of data involved in such solutions (both acquired 
and generated), the focus area (as presented in Table 6), and the degree of "upgrades intelligence" [26]. Our SLR findings agree with previous literature reviews [10,13]; by making a connection between digital solutions and the complete transformation roadmap, SMEs can better plan resource allocation, envision the capabilities development, and understand that each SME has its specific needs and therefore should have its own customized Industry 4.0 vision.

\subsection{Research gap 3: development of resilient production systems under 14.0}

In a sustainable manufacturing ideal model, the design and operation of a manufacturing system must consider numerous factors in the environment and society. The system's effectiveness depends significantly on the sufficiency and promptness of data acquisition, sharing, processing, and utilizing smart things in the system [28]. Besides, to achieve resilience, the system needs to tolerate large and small perturbations during production and still achieve the production goals, e.g., product quality, delivery time, and production cost [55].

It must be stated that proactively implementing resiliency concepts can be a costly strategy for organizations, as they might never be used; this applies for both large and small companies. Therefore, a "one-size-fits-all" approach will not work. Instead, the resilience strategy has to be optimized to a specific environment, in this case, I4.0 manufacturers and SMEs [41]. Resilience is applicable to not only multinationals; the differentiation and winning point over big companies can lie in SMEs' faster implementation.

Standardization among the production environments enables organizations to control complexity and reduce repairs, replacements, and maintenance costs. Diversity within systems minimizes the possibility of cascading system failures. Organizations need to find a balance between their standardized system environment and diversity (ibid.). SMEs need a supporting infrastructure and processes that enable them to quickly acquire, process, and analyze data to reduce their lead times and improve the trustworthiness of the information for better decisionmaking [42].

The results in this SLR showed that the characteristics to be resilient might have been already identified in works like Morisse and Prigge [41]; still, the operationalization of such traits is missing. Further research should focus on a practical resilience approach that considers cost as a highly limiting factor for SMEs. Still, there is a need to improve current deviation management practices by actively integrating digital technologies.

\subsection{Research gap 4: 14.0 as an enabler for sustainability operationalization}

Establishing frameworks and schemes for implementing I4.0 that consider sustainability and a circular economy can save resources and effort in the long run. Such frameworks and schemes can set a threshold toward more sustainable organizations. Nonetheless, there is no standardized deployment model, which makes it essential to understand the specific organization's situation and the changing economic environment. Our findings agree with Garcia-Muiña, GonzálezSánchez, Ferrari, and Settembre-Blundo [49] that circular economy is not a general economic model that ineludibly needs to be adopted to declare sustainable manufacturing operations. In connection to RQ2, we found that SMEs are not exempt from defining a target architecture and developing a roadmap; in the long run, it will allow them to change the organizational structure to become a more flexible, agile, and competitive organization [41]; it is not enough to rely on the implementation of single solutions or I4.0 elements, and there is no shortcut to implement sustainable manufacturing [47]. With the transformation to I4.0, organizations' management and leadership change; SMEs can utilize such change to initiate innovation toward increased sustainability. SMEs' managers need to become more flexible and adaptable with a broader range of knowledge. Empirical research on developing and deploying frameworks that enhance sustainability through I4.0 technologies in SMEs is necessary, with a clear description of SMEs' implementation processes and defined tangible advantages.

\section{Conclusion, recommendations, and limitations of the study}

\subsection{Conclusion and recommendations}

This paper investigated the degree of digitalization in solutions for deviation handling in SMEs and their integration in SMEs' digital transformation. It classified the studies in the form of a conceptual framework to overview the status of research in the area. From the literature review results, five different research streams were identified and classified into five blocks, as in Fig. 3: (1) reviews and perspectives on I4.0; (2) guidelines and frameworks; (3) practical applications in SMEs; (4) deviations and disturbances linked to robustness and resilience under I4.0; and (5) emerging topics. Our SLR results revealed no similar study that included deviation management when analyzing SMEs in the I4.0 context. Therefore, Fig. 3 can serve as a guide map for both researchers and practitioners. A map to (1) determine the research status in developing support knowledge for SMEs; and (2) place attention on underdeveloped areas of I4.0 in 
the SME context, i.e., frameworks that integrate sustainability, resilience, and data analytics to implement I4.0 in SMEs.

The present study is under the umbrella of I4.0 during the past ten years; we expected a limited number of studies with a different distribution than that described in the five categorization blocks; for instance, recognized I4.0 frameworks such as RAMI and the acatech model [24, 25] did not appear in the search results. The most significant block was practical applications, which tells us that the majority of small companies are directly going into action, applying single elements of I4.0 without a clear understanding of the concepts yet. It was demonstrated that I4.0 is not a predefined concept filled with the same content, i.e., small single components/solutions. Companies seem to opt to select those digitalization solutions most suited to their context, to the best of their knowledge and technical capabilities. It can be arguable whether that is the correct path; however, one can highlight the constructive side of learning from experimentations. The "learn by doing" approach seems preferred by SME companies. As seen in Table 6, all the research papers looked at cost and investment as factors that either triggered or hindered the development. The fact that SMEs have small investment possibilities increases the importance of developing a roadmap/planning path to gradually transition and expand their digital elements rather than waiting for a complete conversion investment that could potentially not happen.

The study's findings have implications for SME managers and practitioners, which relate directly to our RQ2. Managers can use the gaps discussed in Section 6 to define internal priorities and requirements for a successful implementation of I4.0. This type of research should aim to prepare and support SMEs in understanding the implications for a successful I4.0 implementation. We summarize our recommendations as follows:

\section{- For research gap 1: perform data mapping before} any tool/system acquisition. A problem faced by other advanced companies in I4.0 is the large and complex data generated by different systems that might not always be used. Additionally, often those different systems may not be able to communicate between themselves. Companies have to add supplementary tools or features to cover the mismatch of current systems, resulting in higher complexity of data and waste in resources. A recommendation would be to examine the following questions: what do we as an organization possess in terms of data? In which form? How do we currently use such data, and how do we further utilize the existing data? Lastly, would the new digital tool or system improve decision-making and facilitate deviation handling? Reflecting on these questions can lead to a better understanding of internal needs and avoid resource waste.
- For research gap 2: embrace the transition by envisioning the whole journey. A digital shift in most businesses may seem imminent and costly in terms of time and resources; however, anticipating the whole journey allows better assessment, planning, and allocating resources and capabilities to meet a tailored I4.0 vision, even if the resources allow the SME to only digitalize at the ERP level, i.e., ISA 95 level 4 [58].

- For research gaps 3 and 4: a resilient and sustainable production system must be the goal, whether we refer to a big or small company, and I4.0 can provide the means for it. SME managers should consider that future production systems should aim for carbon neutrality while tolerating significant disruptions and still accomplish production objectives. The new technological advancements should persist as support to achieve such a vision while remaining competitive, which means enabling the capability to meet social, environmental, and economic goals.

The findings in this study made clear the premature state of digitalization in solutions for deviation handling in SMEs. However, they highlighted the potential for improvement in deviation handling in different areas of the manufacturing site in the long run (Table 6). The fact that practical applications were the biggest block in the findings denotes the little awareness players have of the enabling benefits of a digital transformation. Therefore, the current article also intends to encourage research that presents I4.0 beyond conceptual frameworks and dedicates effort into depicting systematically how to reach gradual stages in the transition, integrating sustainability, circular economy, and data analytics in the big picture. The present article contributes to setting a research agenda to create knowledge that supports SMEs' deviation handling and digital transformation, pointing out connections and gaps in the research streams.

The current article proposes to prioritize further research on the following:

- Standardization both in conceptualization and in practical applications. The degree of digitalization of each application is hard to assess, as there is no joint agreement on the desired full state that complies with I4.0 (RQ1).

- Empirical work on how digital solutions influence deviation handling's prioritization, decision-making, and production performance. A clear connection between the applications and digital element effects on the deviations and disturbances in SMEs was not identified; further studies need to investigate how the adoption of I4.0 elements impacts the deviation management and control in SMEs (RQ2).

- Resilience under I4.0. Future studies should analyze resilience characteristics within I4.0 scaled to SMEs to 
ensure that companies build their projects and implementations on concrete theoretical bases, even on a limited budget, as is common in SMEs.

- Sustainable ways of working. Studies on the relations between data analytics and sustainability and their role in I4.0 deployment are necessary to present frameworks that facilitate SMEs' transition to sustainable and digital ways of operating.

- Lastly, further research should emphasize utilizing the elements that comply with I4.0 to achieve organizational excellence rather than set it as the ultimate goal.

\subsection{Limitations of the study}

One of the limitations of this paper is selecting only four databases, as there might have existed relevant articles outside those which could have been considered. The literature review comprised a small number of articles related to I4.0 and SMEs, which is not ideal for drawing general conclusions. Still, it was anticipated, given the early stage of the terminology. Contemplating the early stage of the included terminology, at the start of the search, we purposely evaluated a scenario in category 6 . We removed the term SMEs and tried to analyze the relation between I4.0 and sustainability. However, the results did not increase; on the contrary, removing the SMEs term would have misled the objective and target of the study. The period selected imposed a second restriction, given the expected increase in the number of research works that capture the development and testing of digital technologies and applications. This research's results are restricted to a selected number of publications; therefore, additional research that considers other databases should validate it.

Author contribution Z. C.: initiated paper concept, methodology definition, performed SLR, analysis, results, and main writing.

M. B.: methodology review, triangulation, writing review, critical analysis, and supervision.

J. B. H.: methodology review, triangulation, writing review, critical analysis, and supervision.

Funding Open access funding provided by Royal Institute of Technology. The authors, with gratitude, acknowledge the financial support of VINNOVA (Programme Produktion2030) for the project ASPIRE "Automation solutions for production deviation management" reference number 2018-01588.

Data availability Supplementary data and materials are available upon request.

\section{Declarations}

Ethical approval This research follows all ethical standards.
Consent to participate All authors agree.

Consent for publication The authors gave consent for this publication.

Competing interests The authors declare no competing interests.

Open Access This article is licensed under a Creative Commons Attribution 4.0 International License, which permits use, sharing, adaptation, distribution and reproduction in any medium or format, as long as you give appropriate credit to the original author(s) and the source, provide a link to the Creative Commons licence, and indicate if changes were made. The images or other third party material in this article are included in the article's Creative Commons licence, unless indicated otherwise in a credit line to the material. If material is not included in the article's Creative Commons licence and your intended use is not permitted by statutory regulation or exceeds the permitted use, you will need to obtain permission directly from the copyright holder. To view a copy of this licence, visit http://creativecommons.org/licenses/by/4.0/.

\section{References}

1. Bertola P, Teunissen J (2018) Fashion 4.0. Innovating fashion industry through digital transformation. Res J Text Appar 22:352369. https://doi.org/10.1108/RJTA-03-2018-0023

2. Kamble SS, Gunasekaran A, Gawankar SA (2018) Sustainable Industry 4.0 framework: a systematic literature review identifying the current trends and future perspectives. Process Saf Environ Prot 117:408-425. https://doi.org/10.1016/j.psep.2018.05.009

3. Moreno M, Charnley F (2016) Can re-distributed manufacturing and digital intelligence enable a regenerative economy? An integrative literature review. Des Manuf 563-575. https://doi.org/10. 1007/978-3-319-32098-4_48

4. Rüßmann M, Lorenz M, Gerbert P et al (2015) Industry 4.0: the future of productivity and growth in Manufacturing Industries. The Boston Consulting Group

5. Lee J, Bagheri B, Kao HA (2015) A cyber-physical systems architecture for Industry 4.0-based manufacturing systems. Manuf Lett 3:18-23. https://doi.org/10.1016/j.mfglet.2014.12.001

6. Brettel M, Friederichsen N, Keller M, Rosenberg M (2014) How virtualization, decentralization and Network Building Change the Manufacturing Landscape: An Industry 4.0 Perspective. 8:37-44

7. Dallasega P, Rojas RA, Rauch E, Matt DT (2017) Simulation based validation of Supply Chain effects through ICT enabled Real-time-capability in ETO Production Planning. Procedia Manuf 11:846-853. https://doi.org/10.1016/j.promfg.2017.07.187

8. Wieland M, Hirmer P, Steimle F et al (2016) Towards a rule-based manufacturing integration assistant. Procedia CIRP 57:213-218. https://doi.org/10.1016/j.procir.2016.11.037

9. Gomes RL, Rigley M, Bacon D et al (2018) Cloud manufacturing as a sustainable process manufacturing route. J Manuf Syst 47:53-68. https://doi.org/10.1016/j.jmsy.2018.03.005

10. Mittal S, Khan MA, Romero D, Wuest T (2018) A critical review of smart manufacturing \& Industry 4.0 maturity models : implications for small and medium-sized enterprises ( SMEs ). J Manuf Syst. https://doi.org/10.1016/j.jmsy.2018.10.005

11. Maier J (2017) Made Smarter review. UK Government. Dep. Business, Energy Ind. Strategy. Available from: https://www.gov.uk/ government/publications/made-smarter-review

12. Li W, Liu K, Belitski M et al (2016) e-Leadership through strategic alignment: an empirical study of small- and medium-sized enterprises in the digital age. J Inf Technol 31:185-206. https:// doi.org/10.1057/jit.2016.10 
13. Moeuf A, Pellerin R, Lamouri S et al (2018) The industrial management of SMEs in the era of Industry 4.0. Int J Prod Res 56:1118-1136. https://doi.org/10.1080/00207543.2017.1372647

14. Juran J, Godfrey A (1998) Juran's quality handbook. 5th edition. McGraw-Hill

15. Islam A, Tedford D (2012) Risk determinants of small and medium-sized manufacturing enterprises (SMEs) - an exploratory study in New Zealand. J Ind Eng Int 8, 12. https://doi.org/10. 1186/2251-712X-8-12

16. Galaske N, Strang D, Anderl R (2015) Process deviations in cyberphysical production systems. In: Proceedings of the World Congress on Engineering and Computer Science 2015, WCECS 2015. San Francisco, USA, pp 1035-1040

17. Bokrantz J, Skoogh A, Ylipää T, Stahre J (2016) Handling of production disturbances in the manufacturing industry. $\mathrm{J}$ Manuf Technol Manag 27:1054-1075. https://doi.org/10.1108/ JMTM-02-2016-0023

18. Martínez-Olvera C, Mora-Vargas J (2018) A max-plus algebra approach to study time disturbance propagation within a robustness improvement context. Math Probl Eng 1-18. https://doi.org/ $10.1155 / 2018 / 1932361$

19. Rüttimann BG, Stöckli MT (2016) Lean and Industry 4.0-twins, partners, or contenders? A due clarification regarding the supposed clash of two production systems. J Serv Sci Manag 09:485500. https://doi.org/10.4236/jssm.2016.96051

20. Buer SV, Strandhagen JO, Chan FTS (2018) The link between industry 4.0 and lean manufacturing: mapping current research and establishing a research agenda. Int J Prod Res 56:2924-2940. https://doi.org/10.1080/00207543.2018.1442945

21. Piccarozzi M, Aquilani B, Gatti C (2018) Industry 4.0 in management studies: a systematic literature review. Sustain 10:1-24. https://doi.org/10.3390/su10103821

22. Machado CG, Winroth MP, Ribeiro da Silva EHD (2019) Sustainable manufacturing in Industry 4.0: an emerging research agenda. Int J Prod Res 58:1462-1484. https://doi.org/10.1080/00207543. 2019.1652777

23. Hofmann E, Rüsch M (2017) Industry 4.0 and the current status as well as future prospects on logistics. Comput Ind 89:23-34. https://doi.org/10.1016/j.compind.2017.04.002

24. Schuh G, Anderl R, Gausemeier J, et al (2017) Industrie 4.0 Maturity Index. Managing the Digital Transformation of Companies (acatech STUDY). https://www.acatech.de/wp-content/ uploads/2018/03/acatech_STUDIE_Maturity_Index_eng_WEB. pdf. Accessed 21 Jul 2020

25. Plattform Industrie 4.0 (2015) RAMI4.0 - a reference framework for digitalisation. In: Plattf. Ind. 4.0. www.plattform-i40.de/I40/ Redaktion/EN/Downloads/Publikation/rami40-an-introduction. pdf?_blob=publicationFile\&v=4. Accessed 21 Jul 2020

26. Wang Z, Shou M, Wang S et al (2019) An empirical study on the key factors of intelligent upgrade of small and mediumsized enterprises in China. Sustain 11. https://doi.org/10.3390/ su11030619

27. Mukhopadhyay A, Murthy LRD, Arora M et al (2019) PCB inspection in the context of smart manufacturing. Springer Singapore, Singapure, pp 781-791

28. Bi Z, Liu Y, Krider J et al (2018) Real-time force monitoring of smart grippers for Internet of Things (IoT) applications. J Ind Inf Integr 11:19-28. https://doi.org/10.1016/j.jii.2018.02.004

29. Kagermann H (2015) Change through digitization-value creation in the age of Industry 4.0. In: Albach H, Meffert H, Pinkwart ARR (eds) Management of permanent change. Springer Gabler, Wiesbaden, pp 23-48

30. Veres P, Béla I, Christian L (2018) Supply chain optimization in automotive industry: a comparative analysis of evolutionary and swarming heuristics. Lect Notes Mech Eng 9:666-676. https:// doi.org/10.1097/00125480-200207000-00002

31. Lee J, Bagheri B, Jin C (2016) Introduction to cyber manufacturing. Manuf Lett 8:11-15. https://doi.org/10.1016/j.mfglet.2016. 05.002

32. Baheti R, Gill H (2011) Cyber-physical Systems impact. Control Technol 12:161-166

33. Cook DJ, Mulrow CD, Haynes RB, Mcmaster F (1997) Systematic review series series editors: Cynthia Mulrow f MD, MSc Deborah Cook f MD, MSc Systematic Reviews: Synthesis of Best Evidence for Clinical Decisions. Ann Intern Med 126:376-380

34. Smart P, Tranfield D, Denyer D (2003) Towards a methodology for developing evidence-informed management knowledge by means of systematic review. Br J Manag 14:207-222. https://doi. org/10.1111/1467-8551.00375

35. Daudi M, Baalsrud J, Thoben K (2016) Behavioral factors influencing partner trust in logistics collaboration : a review. Logist Res. https://doi.org/10.1007/s12159-016-0146-7

36. Pittaway L, Thorpe R, Holt R, Macpherson A (2005) Using Knowledge within small and medium-sized firms : a systematic review of the evidence. Management 7:257-281. https://doi.org/ 10.1111/j.1468-2370.2005.00116.x

37. Popay J, Rogers A, Williams G (1999) Rationale and standards for the systematic review of qualitative literature. J Compos Mater 33:928-940

38. Säfsten K, Gustavsson M (2020) Research methodology - for engineers and other problem-solvers, 1st ed. Studentlitteratur AB. ISBN: 9789144122304 (print)

39. Ud Din F, Henskens F, Paul D, Wallis M (2018) Agent-oriented smart factory (AOSF): an MAS based framework for SMEs under industry 4.0. Smart Innov Syst Technol 96:44-54. https://doi.org/ 10.1007/978-3-319-92031-3_5

40. Boorla MS, Eifler T, McMahon C, Howard TJ (2018) Product robustness philosophy - a strategy towards zero variation manufacturing (ZVM). Manag Prod Eng Rev 9:3-12. https://doi.org/ $10.24425 / 119520$

41. Morisse M, Prigge C (2017) Design of a business resilience model for Industry 4.0 manufacturers. Twenty-third Americas Conference on Information Systems, Boston, pp. 1-10

42. Dubey R, Gunasekaran A, Childe SJ et al (2019) Empirical investigation of data analytics capability and organizational flexibility as complements to supply chain resilience. Int J Prod Res 1-19. https://doi.org/10.1080/00207543.2019.1582820

43. Ramírez-Correa PE, Grandón EE, Arenas-Gaitán J et al (2019) Explaining performance expectancy of IoT in Chilean SMEs. Lect Notes Bus Inf Process 353:475-486. https://doi.org/10.1007/9783-030-20485-3_37

44. Stock T, Seliger G (2016) Opportunities of sustainable manufacturing in Industry 4.0. Procedia CIRP 40:536-541. https://doi.org/ 10.1016/j.procir.2016.01.129

45. Jones M, Zarzycki L, Murray G (2019) Does industry 4.0 pose a challenge for the sme machine builder? A case study and reflection of readiness for a UK SME. IFIP Adv Inf Commun Technol 530:183-197. https://doi.org/10.1007/978-3-030-05931-6_17

46. Wang XL, Wang L, Bi Z et al (2016) Cloud computing in human resource management (HRM) system for small and medium enterprises (SMEs). Int J Adv Manuf Technol 84:485-496. https://doi. org/10.1007/s00170-016-8493-8

47. Bi ZM, Liu Y, Baumgartner B et al (2015) Reusing industrial robots to achieve sustainability in small and medium-sized enterprises (SMEs). Ind Rob 42:264-273. https://doi.org/10.1108/ IR-12-2014-0441

48. Bi Z, Pomalaza-Ráez C, Singh Z et al (2014) Reconfiguring machines to achieve system adaptability and sustainability: a 
practical case study. J Eng Manuf 228:1676-1688. https://doi. org/10.1177/0954405413519788

49. Garcia-Muiña FE, González-Sánchez R, Ferrari AM, SettembreBlundo D (2018) The paradigms of Industry 4.0 and circular economy as enabling drivers for the competitiveness of businesses and territories: the case of an Italian ceramic tiles manufacturing company. Soc Sci 7(12). https://doi.org/10.3390/socsci7120255

50. Hermann M, Pentek T, Otto B (2016) Design principles for industrie 4.0 scenarios. Proc Annu Hawaii Int Conf Syst Sci 2016March 3928-3937. https://doi.org/10.1109/HICSS.2016.488

51. Powell D, Alfnes E, Strandhagen JO, Dreyer H (2013) The concurrent application of lean production and ERP: towards an ERPbased lean implementation process. Comput Ind 64:324-335

52. Müller JM, Buliga O, Voigt KI (2018) Fortune favors the prepared: How SMEs approach business model innovations in Industry 4.0. Technol Forecast Soc Chang 132:2-17. https://doi.org/10. 1016/j.techfore.2017.12.019

53. Sjödin DR, Parida V, Leksell M, Petrovic A (2018) Smart Factory implementation and process innovation: a preliminary maturity model for leveraging digitalization in manufacturing. Res Technol Manag 61:22-31. https://doi.org/10.1080/08956308.2018.1471277
54. Stoldt J, Trapp TU, Toussaint S et al (2018) Planning for digitalisation in SMEs using tools of the digital factory. Procedia CIRP 72:179-184. https://doi.org/10.1016/j.procir.2018.03.100

55. Mittal S, Khan MA, Romero D, Wuest T (2019) Smart manufacturing: characteristics, technologies and enabling factors. J Eng Manuf 233:1342-1361. https://doi.org/10.1177/0954405417736547

56. Gustavsson M, Wänström C (2009) Assessing information quality in manufacturing planning and control processes. 26:325-340. https://doi.org/10.1108/02656710910950333

57. Manyika J, Chui Brown MBJB et al (2011) Big data: the next frontier for innovation, competition, and productivity. McKinsey Global Institute. Available: https://bigdatawg.nist.gov/pdf/MGI_ big_data_full_report.pdf

58. ISA (2013) ANSI/ISA-95.00.01-2010 Enterprise-Control System Integration. Part 1: Models and Terminology. IEC 62264-1 standard. Available: https://www.isa.org/

Publisher's note Springer Nature remains neutral with regard to jurisdictional claims in published maps and institutional affiliations. 